\title{
One Diophantine inequality with unlike powers of prime variables
}

Wenxu $\mathrm{Ge}^{1 *}$ and Weiping $\mathrm{Li}^{2}$

${ }^{\text {"Correspondence: }}$ gewenxu1982@163.com

1 Department of Mathematics and Information Sciences, North China University of Water Resources and Electric Power, Zhengzhou, 450046, P.R. China

Full list of author information is available at the end of the article

\begin{abstract}
In this paper, we show that if $\lambda_{1}, \lambda_{2}, \lambda_{3}, \lambda_{4}, \lambda_{5}$ are nonzero real numbers not all of the same sign, $\eta$ is real, $0<\sigma<\frac{1}{720}$, and at least one of the ratios $\lambda_{j} / \lambda_{j}(1 \leq i<j \leq 5)$ is irrational, then the inequality $\left|\lambda_{1} p_{1}+\lambda_{2} p_{2}^{2}+\lambda_{3} p_{3}^{3}+\lambda_{4} p_{4}^{4}+\lambda_{5} p_{5}^{5}+\eta\right|<\left(\max _{1 \leq j \leq 5} p_{j}^{j}\right)^{-\sigma}$ has infinite solutions with primes $p_{1}, p_{2}, p_{3}, p_{4}, p_{5}$.
\end{abstract}

MSC: 11D75; 11P55

Keywords: Davenport-Heilbronn method; prime; Diophantine approximation

\section{Introduction}

Diophantine inequalities with integer or prime variables have been considered by many scholars. Recently, Yang and Li in [1] proved that the inequality

$$
\left|\lambda_{1} x_{1}^{2}+\lambda_{2} x_{2}^{3}+\lambda_{3} x_{3}^{4}+\lambda_{4} x_{4}^{5}-p-\frac{1}{2}\right|<\frac{1}{2}
$$

has infinite solutions with natural numbers $x_{1}, x_{2}, x_{3}, x_{4}$ and prime $p$. Using the DavenportHeilbronn method, we establish our result as follows.

Theorem 1.1 Let $\lambda_{1}, \lambda_{2}, \lambda_{3}, \lambda_{4}, \lambda_{5}$ be nonzero real numbers not all of the same sign, $\eta$ is real, $0<\sigma<\frac{1}{720}$, and at least one of the ratios $\lambda_{i} / \lambda_{j}(1 \leq i<j \leq 5)$ is irrational, then the inequality

$$
\left|\lambda_{1} p_{1}+\lambda_{2} p_{2}^{2}+\lambda_{3} p_{3}^{3}+\lambda_{4} p_{4}^{4}+\lambda_{5} p_{5}^{5}+\eta\right|<\left(\max _{1 \leq j \leq 5} p_{j}^{j}\right)^{-\sigma}
$$

has infinite solutions with primes $p_{1}, p_{2}, p_{3}, p_{4}, p_{5}$.

\section{Notation and outline of the proof}

Throughout, we use $p$ to denote a prime number. We denote by $\delta$ a sufficiently small positive number and by $\varepsilon$ an arbitrarily small positive number, not necessarily the same at different occurrences. Constants, both explicit and implicit, in Landau or Vinogradov symbols may depend on $\lambda_{1}, \lambda_{2}, \lambda_{3}, \lambda_{4}, \lambda_{5}$, and $\eta$. We write $e(x)=e^{2 \pi i x}$. We take $X$ to be the basic parameter, a large real integer. Since at least one of the ratios $\lambda_{i} / \lambda_{j}(1 \leq i<j \leq 5)$ is irrational, without loss of generality we may assume that $\lambda_{1} / \lambda_{2}$ is irrational. For the other

(c) $2016 \mathrm{Ge}$ and Li. This article is distributed under the terms of the Creative Commons Attribution 4.0 International License (http://creativecommons.org/licenses/by/4.0/), which permits unrestricted use, distribution, and reproduction in any medium, provided you give appropriate credit to the original author(s) and the source, provide a link to the Creative Commons license, and indicate if changes were made. 
cases, the only difference is in the following intermediate region, and we may deal with the same method in Section 4.

Since $\lambda_{1} / \lambda_{2}$ is irrational, there are infinitely many pairs of integers $q$, $a$ with $\mid \lambda_{1} / \lambda_{2}-$ $a / q \mid \leq q^{-2},(a, q)=1, q>0$, and $a \neq 0$. We choose $q$ to be large in terms of $\lambda_{1}, \lambda_{2}, \lambda_{3}, \lambda_{4}, \lambda_{5}$, $\eta$ and make the following definitions:

$$
\begin{aligned}
& N=q^{2}, \quad L=\log N, \quad 0<\sigma<\frac{\theta}{32}<\frac{1}{720}, \nu=N^{-\sigma}, \quad \tau=N^{-1+\theta}, \\
& P=N^{\theta} L^{-1}, \quad Q=\left(\left|\lambda_{1}\right|^{-1}+\left|\lambda_{2}\right|^{-1}\right) N^{1-\theta}, \quad T_{1}=T_{2}^{2}=T_{3}^{3}=T_{4}^{4}=T_{5}^{5}=N^{\frac{1}{3}} .
\end{aligned}
$$

Let $u$ be a positive real number, we define

$$
\begin{aligned}
& K_{u}(\alpha)=\left(\frac{\sin \pi u \alpha}{\pi \alpha}\right)^{2} \quad(\alpha \neq 0), \quad K_{u}(0)=u^{2}, \\
& F_{k}(\alpha)=\sum_{(\delta N)^{1 / k} \leq p \leq N^{1 / k}} e\left(\lambda_{k} p^{k} \alpha\right) \log p, \quad k=1,2,3,4,5, \\
& I_{k}(\alpha)=\int_{(\delta N)^{1 / k}}^{N^{1 / k}} e\left(\lambda_{k} y^{k} \alpha\right) \mathrm{d} y, \quad k=1,2,3,4,5, \\
& J_{k}(\alpha)=\sum_{\substack{|\gamma| \leq T_{k} \\
\beta \geq \frac{2}{3}}} \sum_{\delta N<n \leq N} n^{-1+\rho / k} e\left(\lambda_{k} \alpha n\right), \quad k=1,2,3,4,5,
\end{aligned}
$$

where $\rho=\beta+i \gamma(\beta, \gamma$ real $)$ is a typical non-trivial zero of the Riemann Zeta function.

It follows from (2.3) that

$$
K_{u}(\alpha) \ll \min \left(u^{2},|\alpha|^{-2}\right), \quad \int_{-\infty}^{+\infty} e(\alpha y) K_{u}(\alpha) \mathrm{d} \alpha=\max (0, u-|y|) .
$$

From (2.7) it is clear that

$$
\begin{aligned}
J & :=\int_{-\infty}^{+\infty} \prod_{j=1}^{5} F_{j}(\alpha) e(\alpha \eta) K_{v}(\alpha) \mathrm{d} \alpha \\
& \leq(\log N)^{5} \sum_{\substack{\left|\lambda_{1} p_{1}+\lambda_{2} p_{2}^{2}+\lambda_{3} p_{3}^{3}+\lambda_{4} p_{4}^{4}+\lambda_{5} p_{5}^{5}+\eta\right|<v \\
(\delta N)^{1 / k} \leq p_{k} \leq N^{1 / k}, k=1,2,3,4,5}} 1 \\
& =:(\log N)^{5} \mathcal{N}(N) .
\end{aligned}
$$

Thus we have

$$
\mathcal{N}(N) \geq(\log N)^{-5} J
$$

To estimate $J$, we split the range of infinite integration into three sections, traditional named the neighborhood of the origin $\mathfrak{C}=\{\alpha \in \mathbb{R}:|\alpha| \leq \tau\}$, the intermediate region $\mathfrak{D}=$ $\{\alpha \in \mathbb{R}: \tau \leq|\alpha| \leq P\}$, the trivial region $\mathfrak{c}=\{\alpha \in \mathbb{R}:|\alpha|>P\}$.

To prove Theorem 1.1, we shall establish that

$$
J(\mathfrak{C}) \gg v^{2} N^{\frac{77}{60}}, \quad J(\mathfrak{D})=o\left(v^{2} N^{\frac{77}{60}}\right), \quad J(\mathfrak{c})=o\left(v^{2} N^{\frac{77}{60}}\right)
$$


in Sections 3, 4, and 5, respectively. Thus

$$
\mathcal{N}(N) \gg v^{2}(\log N)^{-5} N^{\frac{77}{60}},
$$

and Theorem 1.1 can be established.

\section{The neighborhood of the origin}

We let

$$
B_{k}(\alpha)=F_{k}(\alpha)-I_{k}(\alpha)+J_{k}(\alpha), \quad k=1,2,3,4,5 .
$$

We use $C$ to denote a positive absolute constant, not necessarily the same one on each occurrence.

Lemma 3.1 We have

$$
B_{k}(\alpha) \ll N^{\frac{2}{3 k}} L^{C}(1+|\alpha| N), \quad k=1,2,3,4,5 .
$$

This is Lemma 7 of Vaughan [2].

Lemma 3.2 For $k=1,2,3,4,5$, we have

$$
\begin{aligned}
& I_{k}(\alpha) \ll N^{\frac{1}{k}} \min \left(1, N^{-1}|\alpha|^{-1}\right), \\
& \int_{-\frac{1}{2}}^{\frac{1}{2}}\left|J_{k}(\alpha)\right|^{2} \mathrm{~d} \alpha \ll N^{\frac{2}{k}-1} \exp \left(-2 L^{-\frac{1}{5}}\right), \\
& \int_{-\frac{1}{2}}^{\frac{1}{2}}\left|I_{k}(\alpha)\right|^{2} \mathrm{~d} \alpha \ll N^{\frac{2}{k}-1}, \\
& \int_{-\tau}^{\tau}\left|B_{k}(\alpha)\right|^{2} \mathrm{~d} \alpha \ll N^{\frac{2}{k}-1} \exp \left(-2 L^{-\frac{1}{5}}\right), \\
& \int_{-\tau}^{\tau}\left|F_{k}(\alpha)\right|^{2} \mathrm{~d} \alpha \ll N^{\frac{2}{k}-1} .
\end{aligned}
$$

Proof The inequality (3.6) follows from (2.1) and Lemma 3.1. The others are similar to Lemma 8 of Vaughan [2].

Lemma 3.3 We have

$$
\int_{\mathfrak{C}}\left|\prod_{i=1}^{5} F_{i}(\alpha)-\prod_{i=1}^{5} I_{i}(\alpha)\right| K_{\nu}(\alpha) \mathrm{d} \alpha \ll v^{2} N^{\frac{77}{60}} \exp \left(-L^{-\frac{1}{5}}\right) .
$$

Proof Note that

$$
\begin{aligned}
& \prod_{i=1}^{5} F_{i}(\alpha)-\prod_{i=1}^{5} I_{i}(\alpha) \\
& \quad=\left(F_{1}(\alpha)-I_{1}(\alpha)\right) \prod_{i=2}^{5} F_{i}(\alpha)+I_{1}(\alpha)\left(F_{2}(\alpha)-I_{2}(\alpha)\right) \prod_{i=3}^{5} F_{i}(\alpha)
\end{aligned}
$$




$$
\begin{aligned}
& +I_{1}(\alpha) I_{2}(\alpha)\left(F_{3}(\alpha)-I_{3}(\alpha)\right) F_{4}(\alpha) F_{5}(\alpha)+\prod_{i=1}^{3} I_{i}(\alpha)\left(F_{4}(\alpha)-I_{4}(\alpha)\right) F_{5}(\alpha) \\
& +\prod_{i=1}^{4} I_{i}(\alpha)\left(F_{5}(\alpha)-I_{5}(\alpha)\right) .
\end{aligned}
$$

Then by (2.7), (3.1), Lemma 3.2,

$$
\begin{aligned}
& \int_{\mathfrak{C}}\left|\left(F_{1}(\alpha)-I_{1}(\alpha)\right) \prod_{i=2}^{5} F_{i}(\alpha)\right| K_{\nu}(\alpha) \mathrm{d} \alpha \\
& \ll v^{2} N^{\frac{47}{60}} \int_{-\tau}^{\tau}\left|\left(B_{1}(\alpha)-J_{1}(\alpha)\right) F_{2}(\alpha)\right| \mathrm{d} \alpha \\
& \ll v^{2} N^{\frac{47}{60}}\left(\int_{-\tau}^{\tau}\left|\left(B_{1}(\alpha)-J_{1}(\alpha)\right)\right|^{2} \mathrm{~d} \alpha\right)^{\frac{1}{2}}\left(\int_{-\tau}^{\tau}\left|F_{2}(\alpha)\right|^{2} \mathrm{~d} \alpha\right)^{\frac{1}{2}} \\
& \ll v^{2} N^{\frac{47}{60}}\left(\int_{-\tau}^{\tau}\left(\left|\left(\left.B_{1}(\alpha)\right|^{2}+\mid J_{1}(\alpha)\right)\right|^{2}\right) \mathrm{d} \alpha\right)^{\frac{1}{2}} \\
& \ll v^{2} N^{\frac{77}{60}} \exp \left(-L^{-\frac{1}{5}}\right) .
\end{aligned}
$$

The other cases are similar, and the proof of Lemma 3.3 is completed.

Lemma 3.4 We have

$$
\int_{|\alpha|>\tau}\left|\prod_{i=1}^{5} I_{i}(\alpha)\right| K_{\nu}(\alpha) \mathrm{d} \alpha \ll v^{2} N^{\frac{77}{60}-4 \theta} .
$$

It follows from (2.7) and (3.3).

\section{Lemma 3.5 We have}

$$
\int_{-\infty}^{+\infty} \prod_{j=1}^{5} I_{j}(\alpha) e(\alpha \eta) K_{v}(\alpha) \mathrm{d} \alpha \gg v^{2} N^{\frac{77}{60}}
$$

Proof To prove (3.10), we write the left side as

$$
\int_{\delta N}^{N} \int_{(\delta N)^{\frac{1}{2}}}^{N^{\frac{1}{2}}} \cdots \int_{(\delta N)^{\frac{1}{5}}}^{N^{\frac{1}{5}}} \int_{-\infty}^{+\infty} e\left(\alpha\left(\eta+\sum_{j=1}^{5} \lambda_{j} y_{j}^{j}\right)\right) K_{v}(\alpha) \mathrm{d} \alpha \mathrm{d} y_{1} \mathrm{~d} y_{2} \cdots \mathrm{d} y_{5},
$$

which, by (2.7), is

$$
\int_{\delta N}^{N} \int_{(\delta N)^{\frac{1}{2}}}^{N^{\frac{1}{2}}} \cdots \int_{(\delta N)^{\frac{1}{5}}}^{N^{\frac{1}{5}}} \max \left(0, v-\left|\eta+\sum_{j=1}^{5} \lambda_{j} y_{j}^{j}\right|\right) \mathrm{d} y_{1} \mathrm{~d} y_{2} \cdots \mathrm{d} y_{5} .
$$

We let $z_{k}=y_{k}^{k}, k=1,2,3,4,5$, then the integral (3.11) can be written as

$$
\frac{1}{120} \int_{\delta N}^{N} \cdots \int_{\delta N}^{N} z_{2}^{-\frac{1}{2}} z_{3}^{-\frac{2}{3}} z_{4}^{-\frac{3}{4}} z_{5}^{-\frac{4}{5}} \max \left(0, v-\left|\eta+\sum_{j=1}^{5} \lambda_{j} z_{j}\right|\right) \mathrm{d} z_{1} \cdots \mathrm{d} z_{5} .
$$


Since $\lambda_{1}, \lambda_{2}, \lambda_{3}, \lambda_{4}$, and $\lambda_{5}$ are not all of the same sign, we may assume without loss of generality that $\lambda_{1}<0, \lambda_{2}>0$. Consider the region

$$
\mathcal{B}=\left\{\left(z_{2}, z_{3}, z_{4}, z_{5}\right): \delta^{\frac{1}{2}} N \leq z_{2} \leq 2 \delta^{\frac{1}{2}} N, \delta N \leq z_{j} \leq 2 \delta N(j=3,4,5)\right\} .
$$

Then, for $\delta$ sufficiently small and large $N$, whenever $\left(z_{2}, z_{3}, z_{4}, z_{5}\right) \in \mathcal{B}$ one has

$$
2 \delta N<-\left(\lambda_{2} z_{2}+\lambda_{3} z_{3}+\lambda_{4} z_{4}+\lambda_{5} z_{5}\right) \lambda_{1}^{-1}<\frac{1}{2} N
$$

and so every $z_{1}$ with $\left|\lambda_{1} z_{1}+\cdots+\lambda_{5} z_{5}+\eta\right| \leq \frac{1}{2} v$ satisfies $\delta N<z_{1}<N$. Therefore the integral (3.12) is greater than

$$
\frac{1}{480} v^{2} \int_{\mathcal{B}} z_{2}^{-\frac{1}{2}} z_{3}^{-\frac{2}{3}} z_{4}^{-\frac{3}{4}} z_{5}^{-\frac{4}{5}} \mathrm{~d} z_{2} \mathrm{~d} z_{3} \mathrm{~d} z_{4} \mathrm{~d} z_{5} \gg v^{2} N^{\frac{77}{60}}
$$

This completes the proof of Lemma 3.5.

Together with Lemmas 3.3, 3.4, 3.5, we have

$$
J(\mathfrak{C})=\int_{\mathfrak{C}} \prod_{j=1}^{5} F_{j}(\alpha) e(\alpha \eta) K_{v}(\alpha) \mathrm{d} \alpha \gg v^{2} N^{\frac{77}{60}} .
$$

\section{The intermediate region}

Lemma 4.1 We have

$$
\begin{aligned}
& \int_{-\infty}^{+\infty}\left|F_{j}(\alpha)\right|^{2^{j}} K_{v}(\alpha) \mathrm{d} \alpha \ll N^{\frac{2^{j}}{j}-1+\varepsilon}, \quad j=2,3,4,5, \\
& \int_{-\infty}^{+\infty}\left|F_{1}(\alpha)\right|^{2} K_{v}(\alpha) \mathrm{d} \alpha \ll N L .
\end{aligned}
$$

Proof By (2.7), we have

$$
\begin{aligned}
& \int_{-\infty}^{+\infty}\left|F_{2}(\alpha)\right|^{4} K_{\nu}(\alpha) \mathrm{d} \alpha \\
& =\sum_{(\delta N)^{\frac{1}{2}} \leq p_{1}, p_{2}, p_{3}, p_{4} \leq N^{\frac{1}{2}}} \prod_{i=1}^{4} \log p_{i} \max \left(0, v-\left|\lambda_{2}\left(p_{1}^{2}+p_{2}^{2}-p_{3}^{2}-p_{4}^{2}\right)\right|\right) \\
& \quad \ll L^{4} \sum_{(\delta N)^{\frac{1}{2}} \leq p_{1}, p_{2}, p_{3}, p_{4} \leq N^{\frac{1}{2}}} \max \left(0, v-\left|\lambda_{2}\left(p_{1}^{2}+p_{2}^{2}-p_{3}^{2}-p_{4}^{2}\right)\right|\right) .
\end{aligned}
$$

Since $N$ is large, $\left|\lambda_{2}\left(p_{1}^{2}+p_{2}^{2}-p_{3}^{2}-p_{4}^{2}\right)\right|<v$ if and only if $p_{1}^{2}+p_{2}^{2}=p_{3}^{2}+p_{4}^{2}$. Thus, by Hua's inequality,

$$
\int_{-\infty}^{+\infty}\left|F_{2}(\alpha)\right|^{4} K_{v}(\alpha) \mathrm{d} \alpha \ll v N^{1+\varepsilon} .
$$

The proofs of the cases $j=3,4,5$ and (4.2) are similar. 
Lemma 4.2 We have

$$
\int_{-\infty}^{+\infty}\left|F_{2}(\alpha)\right|^{2}\left|F_{4}(\alpha)\right|^{4} K_{v}(\alpha) \mathrm{d} \alpha \ll \nu N^{1+\varepsilon} .
$$

Proof By (2.7), we have

$$
\begin{aligned}
& \int_{-\infty}^{+\infty}\left|F_{2}(\alpha)\right|^{2}\left|F_{4}(\alpha)\right|^{4} K_{v}(\alpha) \mathrm{d} \alpha \\
& \ll L^{6} \sum_{\substack{(\delta N)^{\frac{1}{2}} \leq p_{1}, p_{2} \leq N^{\frac{1}{2}} \\
(\delta N)^{\frac{1}{4}} \leq p_{3}, p_{4}, p_{5}, p_{6} \leq N^{\frac{1}{4}}}} \max \left(0, v-\left|\lambda_{2}\left(p_{1}^{2}-p_{2}^{2}\right)-\lambda_{4}\left(p_{3}^{4}+p_{4}^{4}-p_{5}^{4}-p_{6}^{4}\right)\right|\right) \\
& \ll v L^{6} R(N),
\end{aligned}
$$

where $R(N)$ is the number of the solutions of the equation

$$
\begin{aligned}
& \lambda_{2}\left(p_{1}^{2}-p_{2}^{2}\right)=\lambda_{4}\left(p_{3}^{4}+p_{4}^{4}-p_{5}^{4}-p_{6}^{4}\right), \\
& (\delta N)^{\frac{1}{2}} \leq p_{1}, p_{2} \leq N^{\frac{1}{2}}, \quad(\delta N)^{\frac{1}{4}} \leq p_{3}, p_{4}, p_{5}, p_{6} \leq N^{\frac{1}{4}} .
\end{aligned}
$$

Then we have

$$
R(N) \ll N^{\frac{1}{2}} \sum_{\substack{(\delta N)^{\frac{1}{4}} \leq p_{3}, p_{4}, p_{5}, p_{6} \leq N^{\frac{1}{4}} \\ p_{3}^{4}+p_{4}^{4}-p_{5}^{4}-p_{6}^{4}=0}} 1+\sum_{\substack{(\delta N)^{\frac{1}{4}} \leq p_{3}, p_{4}, p_{5}, p_{6} \leq N^{\frac{1}{4}} \\ p_{3}^{4}+p_{4}^{4}-p_{5}^{4}-p_{6}^{4} \neq 0}} d\left(\left|p_{3}^{4}+p_{4}^{4}-p_{5}^{4}-p_{6}^{4}\right|\right),
$$

where $d(n)$ is the divisor function. Now (4.3) follows from [3], (2.1).

Lemma 4.3 ([4]) Suppose that $(a, q)=1,|\alpha-a / q| \leq q^{-2}$, then

$$
\sum_{1 \leq p \leq X}(\log p) e(p \alpha) \ll(\log X)^{5}\left(X^{1 / 2} q^{1 / 2}+X^{4 / 5}+X q^{-1 / 2}\right)
$$

Lemma 4.4 ([5]) Suppose that $(a, q)=1,|\alpha-a / q| \leq q^{-2}, \phi(x)=\alpha x^{k}+\alpha_{1} x^{k-1}+\cdots+\alpha_{k-1} x+$ $\alpha_{k}(k \geq 2)$, then

$$
\sum_{1 \leq p \leq X}(\log p) e(\phi(p)) \ll X^{1+\varepsilon}\left(q^{-1}+X^{-1 / 2}+q X^{-k}\right)^{4^{1-k}}
$$

Lemma 4.5 For $\tau<|\alpha| \leq P$, we have

$$
V(\alpha):=\min \left(F_{1}(\alpha), F_{2}(\alpha)^{2}\right) \ll N^{1-\frac{\theta}{2}+\varepsilon} .
$$

Proof Let $\tau<|\alpha| \leq P$, we choose $a_{j}, q_{j}(j=1,2)$ so that $\left|\lambda_{j} \alpha-a_{j} / q_{j}\right| \leq Q^{-1} q_{j}^{-1}$ with $\left(a_{j}, q_{j}\right)=1$ and $1 \leq q_{j} \leq Q$. By the method of Davenport and Heilbronn (see Lemma 11 of [6]), we have $\max \left(q_{1}, q_{2}\right) \geq P$. Then Lemma 4.5 follows from Lemmas 4.3 and 4.4 . 
Lemma 4.6 We have

$$
J(\mathfrak{D})=\int_{\mathfrak{D}} \prod_{j=1}^{5} F_{j}(\alpha) e(\alpha \eta) K_{v}(\alpha) \mathrm{d} \alpha \ll v^{2} N^{\frac{77}{60}-\left(\frac{\theta}{32}-\sigma\right)+\varepsilon} .
$$

Proof By Lemmas 4.1, 4.2, 4.5, and Hölder's inequality, we have

$$
\begin{aligned}
& \int_{\mathfrak{D}} \mid \prod_{j=1}^{5} F_{j}(\alpha) e(\alpha \eta) K_{v}(\alpha) \mid \mathrm{d} \alpha \\
& \ll V(\alpha)^{\frac{1}{16}} \int_{-\infty}^{+\infty}\left|\left(F_{1}(\alpha)^{\frac{15}{16}} F_{2}(\alpha)+F_{1}(\alpha) F_{2}(\alpha)^{\frac{7}{8}}\right) \prod_{j=3}^{5} F_{j}(\alpha)\right| K_{v}(\alpha) \mathrm{d} \alpha \\
& \ll V(\alpha)^{\frac{1}{16}}\left(\int_{-\infty}^{+\infty}\left|F_{1}(\alpha)\right|^{2} K_{v}(\alpha) \mathrm{d} \alpha\right)^{\frac{15}{32}}\left(\int_{-\infty}^{+\infty}\left|F_{2}(\alpha)\right|^{4} K_{v}(\alpha) \mathrm{d} \alpha\right)^{\frac{1}{8}} \\
& \quad \times\left(\int_{-\infty}^{+\infty}\left|F_{2}(\alpha)^{2} F_{4}(\alpha)^{4}\right| K_{v}(\alpha) \mathrm{d} \alpha\right)^{\frac{1}{4}}\left(\int_{-\infty}^{+\infty}\left|F_{3}(\alpha)\right|^{8} K_{v}(\alpha) \mathrm{d} \alpha\right)^{\frac{1}{8}} \\
& \quad \times\left(\int_{-\infty}^{+\infty}\left|F_{5}(\alpha)\right|^{32} K_{v}(\alpha) \mathrm{d} \alpha\right)^{\frac{1}{32}}+V(\alpha)^{\frac{1}{16}}\left(\int_{-\infty}^{+\infty}\left|F_{1}(\alpha)\right|^{2} K_{v}(\alpha) \mathrm{d} \alpha\right)^{\frac{1}{2}} \\
& \quad \times\left(\int_{-\infty}^{+\infty}\left|F_{2}(\alpha)\right|^{4} K_{v}(\alpha) \mathrm{d} \alpha\right)^{\frac{3}{32}}\left(\int_{-\infty}^{+\infty}\left|F_{2}(\alpha)^{2} F_{4}(\alpha)^{4}\right| K_{v}(\alpha) \mathrm{d} \alpha\right)^{\frac{1}{4}} \\
& \quad \times\left(\int_{-\infty}^{+\infty}\left|F_{3}(\alpha)\right|^{8} K_{v}(\alpha) \mathrm{d} \alpha\right)^{\frac{1}{8}}\left(\int_{-\infty}^{+\infty}\left|F_{5}(\alpha)\right|^{32} K_{v}(\alpha) \mathrm{d} \alpha\right)^{\frac{1}{32}} \\
& \ll v N^{\frac{77}{60}-\frac{\theta}{32}+\varepsilon} \ll v^{2} N^{\frac{77}{60}-\left(\frac{\theta}{32}-\sigma\right)+\varepsilon} .
\end{aligned}
$$

\section{The trivial region}

Lemma 5.1 Let $G(\alpha)=\sum e\left(\alpha f\left(x_{1}, \ldots, x_{m}\right)\right)$, where $f$ is any real function and the summation is over any finite set of values of $x_{1}, \ldots, x_{m}$. Then, for any $A>4$, we have

$$
\int_{|\alpha|>A}|G(\alpha)|^{2} K_{v}(\alpha) \mathrm{d} \alpha \leq \frac{16}{A} \int_{-\infty}^{+\infty}|G(\alpha)|^{2} K_{v}(\alpha) \mathrm{d} \alpha .
$$

This is Lemma 2 of [7].

Lemma 5.2 We have

$$
J(\mathfrak{c})=\int_{\mathfrak{c}} \prod_{j=1}^{5} F_{j}(\alpha) e(\alpha \eta) K_{\nu}(\alpha) \mathrm{d} \alpha \ll v^{2} N^{\frac{77}{60}-(\theta-\sigma)+\varepsilon} .
$$

Proof By Lemmas 4.1, 4.2, 5.1, and Hölder's inequality, we have

$$
\begin{aligned}
\int_{\mathfrak{c}} \mid & \prod_{j=1}^{5} F_{j}(\alpha) e(\alpha \eta) K_{v}(\alpha) \mid \mathrm{d} \alpha \\
& \ll \frac{1}{P} \int_{-\infty}^{+\infty} \prod_{j=1}^{5}\left|F_{j}(\alpha)\right| K_{\nu}(\alpha) \mathrm{d} \alpha
\end{aligned}
$$




$$
\begin{aligned}
& \ll \frac{1}{P} \max \left(\left|F_{5}(\alpha)\right|\right)\left(\int_{-\infty}^{+\infty}\left|F_{1}(\alpha)\right|^{2} K_{v}(\alpha) \mathrm{d} \alpha\right)^{\frac{1}{2}}\left(\int_{-\infty}^{+\infty}\left|F_{2}(\alpha)\right|^{4} K_{\nu}(\alpha) \mathrm{d} \alpha\right)^{\frac{1}{8}} \\
& \quad \times\left(\int_{-\infty}^{+\infty}\left|F_{2}(\alpha)^{2} F_{4}(\alpha)^{4}\right| K_{v}(\alpha) \mathrm{d} \alpha\right)^{\frac{1}{4}}\left(\int_{-\infty}^{+\infty}\left|F_{3}(\alpha)\right|^{8} K_{\nu}(\alpha) \mathrm{d} \alpha\right)^{\frac{1}{8}} \\
& \ll v N^{\frac{77}{60}-\theta+\varepsilon} \ll v^{2} N^{\frac{77}{60}-(\theta-\sigma)+\varepsilon} .
\end{aligned}
$$

\section{Competing interests}

The authors declare that they have no competing interests.

\section{Authors' contributions}

All authors contributed equally to the writing of this paper. All authors read and approved the final manuscript.

\section{Author details}

'Department of Mathematics and Information Sciences, North China University of Water Resources and Electric Power, Zhengzhou, 450046, P.R. China. ${ }^{2}$ Department of Mathematics and Information Science, Henan University of Economics and Law, Zhengzhou, 450046, P.R. China.

\section{Acknowledgements}

This work is supported by the National Natural Science Foundation of China (Grant Nos. 11371122, 11471112), the Innovation Scientists and Technicians Troop Construction Projects of Henan Province (China), the Research Foundation of North China University of Water Conservancy and Electric Power (No. 201084).

Received: 18 November 2015 Accepted: 19 January 2016 Published online: 28 January 2016

\section{References}

1. Yang, TQ, Li, WP: One Diophantine inequality with integer and prime variables. J. Inequal. Appl. 2015, 293 (2015)

2. Vaughan, RC: Diophantine approximation by prime numbers, II. Proc. Lond. Math. Soc. 28, 385-401 (1974)

3. Brüdern, J, Kawada, K: Ternary problems in additive prime number theory. Dev. Math. 6, $39-91$ (2002)

4. Vaughan, RC: The Hardy-Littlewood Method. Cambridge University Press, Cambridge (1997)

5. Harman, G: Trigonometric sums over primes I. Mathematika 28, 249-254 (1981)

6. Vaughan, RC: Diophantine approximation by prime numbers, I. Proc. Lond. Math. Soc. 28, 373-384 (1974)

7. Davenport, H, Roth, KF: The solubility of certain Diophantine inequalities. Mathematika 2, 81-96 (1955)

\section{Submit your manuscript to a SpringerOpen ${ }^{\circ}$ journal and benefit from:}

- Convenient online submission

Rigorous peer review

- Immediate publication on acceptance

- Open access: articles freely available online

- High visibility within the field

- Retaining the copyright to your article 